\title{
Activités
}

\section{La fidélisation de nouveaux dans une entreprise du BTP}

Approche ergonomique des enjeux et des déterminants

Developing loyalty among newcomers in a construction company: ergonomics approach to issues and determining factors

La fidelización de los nuevos empleados en una empresa constructora : Enfoque ergonómico de los desafíos y de los determinantes

\section{Corinne Gaudart, Catherine Delgoulet et Karine Chassaing}

\section{OpenEdition \\ Journals}

Édition électronique

URL : http://journals.openedition.org/activites/2013

DOI : 10.4000/activites.2013

ISSN : 1765-2723

\section{Éditeur}

ARPACT - Association Recherches et Pratiques sur les ACTivités

\section{Référence électronique}

Corinne Gaudart, Catherine Delgoulet et Karine Chassaing, « La fidélisation de nouveaux dans une entreprise du BTP », Activités [En ligne], 5-2 | octobre 2008, mis en ligne le 15 octobre 2008, consulté le 01 mai 2019. URL : http://journals.openedition.org/activites/2013; DOI : 10.4000/activites.2013

\section{cc)}

Activités est mis à disposition selon les termes de la licence Creative Commons Attribution - Pas d'Utilisation Commerciale - Pas de Modification 4.0 International. 


\title{
La fidélisation de nouveaux dans une entreprise du BTP: Approche ergonomique des enjeux et des déterminants
}

\author{
Corinne Gaudart \\ CNRS/CREAPT/CEE \\ « Le Descartes I » 29, Promenade Michel Simon - 93166 Noisy-Le-Grand Cedex. \\ corinne.gaudart@cee.enpc.fr
}

\section{Catherine Delgoulet}

EA 4070 «Ergonomie, Comportement et Interactions », Université Paris Descartes - 45, rue des Saints-Pères 75270 PARIS cedex 06. catherine.delgoulet@univ-paris5.fr

\section{Karine Chassaing}

CREAPT/CEE

« Le Descartes I » 29, Promenade Michel Simon - 93166 Noisy-Le-Grand Cedex. karine.chassaing@wanadoo.fr

\begin{abstract}
Developing loyalty among newcomers in a construction company: ergonomics approach to issues and determining factors. The retirement of present and future baby-boomers is an opportunity for enterprises to renew their labour force. Moreover, many businesses find it hard to retain newly hired employees who are amongst the youngest, despite the current context of unemployment. This could be due to a number of factors which further complicate a problem often regarded in terms of developing loyalty among newcomers, a generational gap and differences in representations. This paper puts forward an ergonomics approach to the situation in a large construction company. Despite the variety of statuses of "new" workers, and the multiple means of retaining them by the welcoming and training they are given, this study shows that "professional gestures" are not the main concern of the HRD or the construction site organisers nor, to a certain extent, of the senior workers themselves. Such a lack of concern leads newcomers to develop opportunist forms of learning by which they must prove themselves, and thus challenges the whole form and contents of these practices whose purpose is to induce loyalty.
\end{abstract}

\section{KEYWORDS}

Inter-generational transmission, loyalty-inducing, learning, professional gestures, construction industry.

Nous entrons dans une période de reconfiguration démographique, dont l'envergure dépendra des volumes d'entrées et de départs de la population active dans les années à venir; elle se caractérise notamment par trois éléments (Blanchet, 2002; Molinié, 2003): des départs en retraite de plus en plus nombreux du fait du vieillissement des générations du baby-boom; un remplacement progressif des générations les plus anciennes par de plus jeunes dont les effectifs restent inférieurs en nombre à celles du baby-boom; une proportion de quinquagénaires qui demeure toujours importante.

Cette nouvelle configuration démographique a pour conséquence de réintroduire une diversité des âges au travail; ce qui déroute certaines entreprises, notamment du secteur industriel, qui avaient jusque-là davantage l'habitude de gérer des amplitudes générationnelles plus resserrées dans les âges 
intermédiaires, caractéristiques du contexte des années 80-90 (Gaudart, Molinié, \& Pueyo, 2006). Cette configuration pose des questions aux entreprises se plaçant dans une démarche anticipatrice de gestion de leur personnel et induit de grosses difficultés pour celles qui se trouvent devant le fait accompli. Au-delà du problème purement arithmétique, ces questions ou difficultés sont de plusieurs ordres:

- Étant donné le vieillissement de la population active (hormis certains secteurs d'activité traditionnellement « jeunes ») et la quasi-disparition des dispositifs de préretraite, quels emplois peut-on proposer aux plus âgés qui n'ont pas encore l'âge de la retraite mais sont parfois usés et envisagent de finir précocement leur carrière (Molinié, 2005a; Hansez, Bertrand, De Keyser, \& Férée, 2005)?

- Comment intègre-t-on des nouveaux, souvent jeunes, dans des entreprises où l'on a une « discontinuité dans les générations » (Molinié, 2001) et à des collectifs de travail fragilisés (déstructurés) par les départs massifs des anciens? Comment dans certains secteurs d'activité (hôpital, construction, hôtellerie-restauration) fidélise-t-on une main-d'œuvre visiblement prompte à quitter ou changer d'emploi ?

- Comment organise-t-on la transmission des savoirs et savoir-faire professionnels, qui peut difficilement être laissée à la discrétion des salariés comme auparavant, étant donné les pertes importantes que suscitent les nombreuses sorties d'activités?

Autrement dit, la prise en compte de la diversité des âges au travail devrait être l'un des axes majeur des entreprises pour accompagner cette transition démographique: tenir compte des spécificités qui se construisent dans le travail avec l'âge et aux différents âges et organiser la transmission entre générations, pour un souci de maintien de la performance.

Face à ces questions, la gestion des ressources humaines (GRH) a des réponses qui visent souvent à proposer une conception différente du parcours professionnel et de l'évaluation - promotion professionnelle. Parmi ces solutions, deux types se sont généralisés dans les entreprises ces 20 dernières années. Le passage de la logique « qualification » à la logique « compétences » s'est traduit par une individualisation des parcours et de l'évaluation des salariés et, comme le rappelle Stroobants (2003), a permis de résoudre un problème de déséquilibre démographique lié aux départs massifs en préretraite. La gestion par les compétences permet de ne plus subordonner la promotion sociale dans les entreprises aux seuls critères de l'ancienneté et du diplôme. Elle donne la possibilité de proposer à des cohortes de jeunes salariés d'ancienneté équivalente des parcours différenciés. Une autre solution retenue consiste à mettre en place des « mesures d'âge » spécifiques aux jeunes ou aux plus âgés (Alexandre-Bailly, Gautié, Guillemard, \& Jolivet, 2004) qui se traduisent par exemple par des règles différentes de rémunération selon les générations ou la mise en place de dispositifs de « fidélisation » des plus jeunes jugés difficiles à garder. Ces dispositifs visent à favoriser la « socialisation organisationnelle » (Almudever, \& Le Blanc, 2006) en :

- Réduisant l'incertitude liée à la nouveauté de la situation (accueil formel du responsable, visite guidée du site, informations « sociales » ou « sécurité », livret d'accueil...);

- Sensibilisant la hiérarchie au rôle de l'articulation de la vie au travail et de la vie hors travail dans l'engagement professionnel (horaires de travail modulables, congés supplémentaires pour un enfant malade...);

- Valorisant les relations interpersonnelles en tant que ressources dans le processus d'intégration (tutorat, mentorat, parrainage...).

Ces différentes démarches placent les travailleurs au centre de la question de fidélisation soit comme « objets » à rendre conformes aux « réalités » de l'entreprise, soit comme « sujets proactifs » détenteurs individuellement ou collectivement des ressources nécessaires et suffisantes à la réussite du processus d'intégration.

Que peut alors dire l'approche ergonomique des enjeux et des déterminants de la fidélisation des nouveaux ? C'est ce que nous souhaiterions développer dans cet article à travers l'étude, dans une grande 
entreprise du BTP, d'une des formes de fidélisation présentée ci-dessus: les modalités d'accueil de nouveaux et leurs conditions. Cette entreprise développe depuis quelques années une politique volontaire en matière de recrutement de nouveaux, de formation et de gestion des ressources humaines pour attirer et fidéliser la main-d'œuvre dans un contexte de pénurie. Toutefois, l'entreprise considère avoir des difficultés à fidéliser ses nouveaux qui la quittent malgré la mise en place de ces dispositifs censés les retenir. Trois secteurs ont été regardés, le bâtiment, les travaux publics de canalisation et le génie civil, avec des conditions d'accueil des nouveaux très variables, du statut d'intérimaire à celui de stagiaire, et avec des modalités de prises en charge plus ou moins formalisées.

\section{1.- Les enjeux de la fidélisation dans le secteur de la construction}

\section{1.- Des chiffres qui posent le problème}

Qu'en est-il des questions de fidélisation dans le secteur de la construction? Les projections d'emplois dans les années à venir mettent l'accent sur un développement important des métiers des services, notamment des services à la personne, mais le secteur de la construction restera un secteur qui recrutera du fait des départs à la retraite (Chardon, Estrade, \& Toutlemonde, 2005). Les entrées en apprentissage sont en progression constante depuis ces dernières années $(+5,8 \%$ en $2004 ;+5,5 \%$ en 2005, soit 260000 nouveaux contrats) dans de nombreux secteurs, et la construction, avec les industries agricoles et alimentaires, figure parmi les secteurs qui recrutent le plus de jeunes sans qualification (Sanchez, 2007), offrant ainsi une « seconde chance » à des élèves en difficulté scolaire pour une insertion professionnelle (Aldeghi, \& Cohen-Scali, 2005). Pourtant, en 2002, $80 \%$ des employeurs du BTP et de l'industrie déclaraient des difficultés à recruter (Chardon, \& Viney, 2003). Malgré un niveau de chômage encore important, cette situation risque de durer, dans la mesure où ces tensions du marché entre offres et demandes auraient, dans la construction, un caractère structurel lié à des conditions de travail qui rendent ces professions peu attractives (Lainé, 2005). Parallèlement, le marché de l'intérim est en expansion et $8,8 \%$ des salariés de la construction étaient intérimaires en 2006 (De Ricardis, 2007). Comment interpréter ces chiffres qui laissent entendre tout à la fois une conjonction favorable au développement de nouvelles carrières dans ce secteur et des difficultés durables pour en renouveler la main-d'œuvre et la stabiliser?

\section{2.- Un secteur connu comme pénible mais valorisant}

Le secteur de la construction est connu pour la pénibilité physique qu'il génère. Dubré, Touranchet, Thérond-Hamidou, Montfort et Derriennic (1996), à partir de l'enquête ESTEV ${ }^{1}$, insistent sur l'importance des douleurs ostéo-articulaires provoquées par la manutention de charges lourdes et les postures pénibles, qui deviennent sélectives au fil du temps. Ces contraintes se trouvent par ailleurs potentiellement renforcées dans les situations de contraintes temporelles (Lasfargues, 2005). Passé 50 ans, pour ceux qui sont restés, l'enquête SVP50² montre que plus de la moitié connaît ou a connu

1. L'enquête «Santé Travail et Vieillissement » est une enquête longitudinale prospective et multicentrique (7 régions françaises) consistant à interroger et à examiner un même échantillon de salariés âgés de 37 à 52 ans à 5 ans d'intervalle (21 378 en 1990 et 18695 en 1995) du point de vue de leur travail et de leur santé pour: évaluer les évolutions avec l'âge des critères de santé (santé objective et santé perçue) en rapport avec le vieillissement; détecter les facteurs professionnels (expositions, contraintes, astreintes) au moment de l'enquête ou dans le passé, mais aussi certaines caractéristiques psychosociales liées à la monotonie, à l'intérêt, à l'autonomie et aux marges de manoeuvre dans le travail susceptibles de modifier les évolutions de la santé avec l'âge. Cette enquête s'est déroulée lors de la visite annuelle médicale.

2. L'enquête « Santé et Vie Professionnelle après 50 ans » est une enquête transversale qui s'est déroulée sur plusieurs mois en 2003. 650 médecins de toutes les régions françaises l'ont réalisée. Chacun d'eux a interrogé tous les salariés de 50 ans et plus lors de la visite médicale annuelle, ou un échantillon aléatoire de ceux-ci. Au total, 11223 questionnaires ont été recueillis. Le questionnaire comporte des informations sur l'itinéraire professionnel du salarié, les principales contraintes auxquelles il est exposé et celles auxquelles il a été exposé par le passé, des appréciations sur l'intérêt et le sens de son travail, sa perception de la retraite et ses intentions en termes d'âges de départ; et divers 
au moins 20 ans de travail physiquement exigeant (Molinié, 2005b). Il semble toutefois qu'il ne faille pas attendre si longtemps pour éprouver des douleurs physiques. Une étude américaine (Merlino, Rosecrance, Anton, \& Cook, 2003) a évalué la prévalence des TMS sur 996 apprentis de la construction, en formation en alternance pendant 4 à 5 ans, en cherchant à déterminer les facteurs causaux dans le champ du travail et de la vie personnelle. Un peu plus de la moitié des apprentis a signalé des douleurs dans le bas du dos, $42 \%$ des douleurs aux poignets et aux mains et $38 \%$ aux genoux. Les troisquarts des apprentis ont signalé des douleurs sur au moins une localisation proposée. Travailler dans la même position sur de longues périodes apparaît comme le facteur causal déterminant. Par ailleurs, ces symptômes, particulièrement les douleurs dans le bas du dos, apparaissent dès la deuxième année d'apprentissage et vont croissants.

La pénibilité du travail dès le stade d'apprentissage peut être mise en lien avec les conditions de l'apprentissage. Cru (1995) évoque combien le bâtiment est un secteur sélectif. Les nombreux départs des plus jeunes peuvent être non seulement attribués aux conditions salariales précaires, mais aussi à la confrontation à la peur. Les apprentis et les intérimaires sont beaucoup plus souvent victimes d'accidents que les autres salariés et les conditions de travail difficiles s'accompagnent d'un risque accru d'accidents (Hamon-Cholet, \& Sandret, 2007). Ceux qui restent dissocient le risque de leur activité au quotidien, sous peine de ne pas pouvoir tenir, en assimilant les idéologies défensives de métier. Aldheghi et Cohen-Scali (2005), dans une enquête portant sur l'orientation et la professionnalisation des jeunes dans le secteur du bâtiment, montrent que parmi les apprentis préparant un CAP, ceux qui ont eu un suivi quotidien, qui ont pu aller à leur rythme et faire un travail varié ont des représentations positives du métier et se voient rester dans le métier plusieurs années. Les autres en ont une image dégradée, particulièrement quand ils ont été soumis à un travail répétitif. En marge de la question de la représentation du métier - ici traitée dans le registre de la psychologie sociale -, ces résultats peuvent laisser supposer des parcours d'apprentissage dont les conditions de travail et d'encadrement hétérogènes auront des conséquences sur l'insertion des nouveaux.

Par ailleurs, c'est un secteur qui possède ses propres spécificités quant aux formes d'investissement de ses opérateurs. Cette même enquête évoque le rôle du contexte familial dans la construction d'une représentation du métier: une première expérience acquise dans le cadre familial et/ou un membre de la famille déjà dans le métier donne des repères positifs au débutant. Le bâtiment devient une valeur familiale, un patrimoine de compétences familiales qui se transmet sur plusieurs générations. Le travail est - selon les auteurs - rattaché à une dimension affective forte. Ces représentations positives se retrouvent à d'autres périodes de la carrière professionnelle sous d'autres formes (Dubré et al., 1996; Molinié, 2005b) et mettent en avant un paradoxe spécifique au secteur de la construction. Malgré les pénibilités du travail, contrairement aux autres métiers ouvriers et à tout âge, ceux du BTP considèrent qu'ils trouvent davantage leur travail varié, une plus grande autonomie dans leur travail, les moyens pour réaliser un travail de qualité et un travail qui permet d'apprendre.

\section{3.- Une prescription du travail « juste-à-temps »}

La construction d'une pratique professionnelle dans le secteur porte elle aussi ses spécificités, notamment parce qu'elle s'inscrit dans un rapport à la prescription marqué par les modes d'organisation du bâtiment; ce que Berthet et Cru (2003) nomment « la prescription déclinée selon les genres ». L'impossibilité de maîtriser toutes les variables du chantier a conduit à faire renoncer les concepteurs à tout prescrire d'emblée. Cette impossibilité de prescrire ne se traduit pas par une absence de prescription, mais par une prescription en juste-à-temps: la prescription est déclinée au fur et à mesure de l'avancement du chantier, dans les différents niveaux hiérarchiques, pour aboutir à la répartition des effectifs et des tâches dans les équipes. Ces réajustements continus ont des conséquences sur l'activité des compagnons dans la nature des compétences qu'ils construisent, mais aussi dans leurs prises de risque (Six, 1997, 1999). Cru et Berthet mettent en avant l'idée qu' "une telle organisation repose sur le présupposé que chacun, à son niveau, connaît son métier et dispose des moyens

aspects de son état de santé, avec leur évolution récente et les gênes éventuelles occasionnées dans le travail. 
pour le faire. [...] Ce mode de prescription table sur l'existence de genres » (p. 88 et 89), au sens où Clot (1999) l'entend: un fond commun de règles et de valeurs de métier qui permettent de travailler ensemble sans que soit nécessaire de respécifier la tâche à chaque fois qu'elle se présente (Clot, \& Faïta, 2000). Cette forme de prescription déclinée selon le genre peut se trouver remise en question par des facteurs de charge de travail (par exemple: tenir des délais malgré les réajustements); mais aussi par des modes de gestion du personnel, quand ils débouchent sur la précarité des personnes: le manque d'habitude des compagnons à travailler avec d'autres fragilise les genres dans leur capacité à anticiper et à agir, et aggrave les effets des conditions de travail et de sécurité des compagnons. Cette précarité peut être due à des recompositions d'équipe d'une tâche à une autre, d'un chantier à un autre, et relève alors de l'organisation du travail; elle peut également être due à la présence d'intérimaires ou de personnel de passage qui dépend de la gestion des ressources humaines.

Duc (2002), avec une approche sociologique, analyse les évolutions du secteur du bâtiment et sa faillite à prescrire dans un modèle taylorien comme débouchant sur une «Organisation du Travail à Prescription Floue »: " ce dispositif organisationnel n'a pas vocation de rationaliser dans le détail les actes de travail mais de favoriser des comportements d'initiative et de prise en charge des variabilités de l'activité » (p. 119). Il est basé sur les principes suivants:

- La prescription floue s'adresse à des équipes d'ouvriers considérés comme des micro-collectifs et a pour caractéristique principale de rester imprécise dans le «tout comment faire ». La détermination du travail à réaliser porte sur les résultats à atteindre et sur le temps d'exécution, mais ne précise pas dans le détail les procédures et les moyens d'y parvenir.

- Ce flou de la prescription est en fait considéré par les organisateurs comme une marge de manœuvre donnant les moyens aux micro-collectifs de faire face à l'imprévisibilité. Elle implique pour cela que l'organisation interne de ces micro-collectifs soit laissée à l'initiative des ouvriers, mais dans un espace limité et sans interférence avec les autres équipes.

- Elle suppose enfin que l'encadrement organise sa propre activité de telle manière qu'il puisse modifier les consignes de planification qu'il a lui-même élaborées.

L'Organisation du Travail à Prescription Floue ressemble à une organisation qui n'aurait pas tranché entre deux modèles opposés (prescription taylorienne vs flexibilité) et qui, au final, se donne l'opportunité de choisir l'un ou l'autre, l'un et l'autre en fonction des événements, sans pour autant prendre la mesure des conséquences pour ceux censés l'appliquer. Une même entreprise du BTP peut ainsi mener de front des politiques GRH contradictoires, basées à la fois sur le désir de recruter dans la durée et sur l'appel à des intérimaires pour réduire les coûts et pallier le turnover. La juxtaposition de ces pratiques n'est pas sans conséquences sur les difficultés entre générations. Duc, au travers de ses visites de chantier, note que l'esprit de solidarité, facteur essentiel dans le travail pour les anciens, est remis en question à deux niveaux : par l'organisation qui ne tranche pas au sujet de la nécessité de collectifs stables, et par les plus jeunes qui expliquent aux anciens que - contrairement à ce qu'il se passait auparavant - ils n'ont pas le temps de se construire sur cette valeur car les contraintes temporelles sont maintenant plus fortes.

Ces auteurs, issus d'horizons disciplinaires différents, partagent plusieurs idées: une forme de prescription qui compte, au bout de la chaîne, sur des compagnons et des collectifs pour mener à bien un travail sans cesse réajusté; des choix organisationnels et des politiques de GRH qui peuvent remettre en cause la stabilité des équipes, pourtant essentielle pour tenir la prescription. Ce sont alors les intérimaires et les nouveaux, souvent jeunes, qui sont ciblés comme facteurs d'instabilité par les anciens et qui - dans la continuité de pensée de Cru et Berthet - seraient donc potentiellement porteurs d'une remise en question du genre professionnel. Pour ces nouveaux, souvent marqués par un rapport à la scolarité et à l'emploi difficile, l'enjeu est de «trouver sa place », dans un contexte où les modalités d'accueil et d'insertion des nouveaux ne sont plus tout à fait les mêmes que pour les anciens ayant connu le compagnonnage (Flamant, 2005). Pourtant, les conditions d'accueil et d'apprentissage semblent déterminantes pour la qualité des conditions de travail dans un métier par ailleurs connu comme pénible et pour la construction d'une représentation positive du métier où l'on se voit durer. 
Qui sont les « nouveaux»? Qu'apprennent-ils ? Comment l'apprennent-ils? Dans quels dispositifs d'accueil? Qui sont les « anciens»? Que transmettent-ils? Comment le transmettent-ils? Quelles sont les incidences de l'organisation du travail sur l'apprentissage? Autant de questions auxquelles nous souhaiterions apporter un éclairage en opérant, par la mise en place d'une approche ergonomique centrée sur l'activité, un double déplacement: d'une représentation GRH de l'accueil et de la fidélisation des nouveaux vers une prise en compte des conditions de l'activité d'apprentissage et de transmission; d'une interprétation psychologisante de caractéristiques générationnelles vers une prise en compte des conditions réelles de travail.

Pour ce faire, le recueil de données de terrain s'est organisé en quatre phases:

1-Une consultation de données générales sur l'âge, le type de contrat et les actions de formation recensés par la Direction Centrale des RH.

2-Des visites de chantier du bâtiment et de travaux publics de canalisation qui accueillaient des nouveaux, dans l'objectif de se familiariser avec les situations de travail et d'élaborer, dans la phase suivante, une grille d'entretien.

3 -Des entretiens réalisés sur le site de formation de l'entreprise à l'occasion de stages destinés à des opérateurs déjà salariés de l'entreprise et concernant les trois branches d'activité. Il s'agissait de stages de perfectionnement proposés à des compagnons ou des stages ciblés sur la fonction de chef d'équipe ou chef de chantier. Ces entretiens, au nombre de 26 , ont duré entre 30 minutes et 1 heure. Ils visaient à reconstituer les parcours de ces salariés, en insistant d'une part sur leur début (modalités d'accueil et de formation) dans le BTP et, d'autre part, sur la manière dont ils accueillent et forment eux-mêmes des nouveaux aujourd'hui. Nous avons également tenté de comprendre en quoi l'organisation et les conditions de travail sur les chantiers ont une incidence sur l'accueil et la formation. Les personnes rencontrées étaient âgées de 22 à 49 ans, avec une expérience de 2 à 33 ans dans le métier.

4-Un suivi, dans le génie civil, de nouveaux intégrés dans un dispositif de formation en alternance relevant d'un partenariat entre l'entreprise et un organisme public de formation pour adultes. Trois coffreurs-bancheurs en formation ont été suivis sur le chantier.

\section{2.- Dans l'entreprise: une gestion floue pour fidéliser}

L'analyse de données générales RH pointe:

- Des lacunes, au regard de la question portée par l'entreprise, dans les outils de gestion des âges, des statuts et des effectifs;

- Des difficultés à attirer des nouveaux malgré la mise en place de dispositifs d'accueil.

\section{1.- Une structure d'âge vieillissante en CDI?}

L'entreprise compte 5748 salariés $^{3}$, avec une forte représentation des salariés de la branche Bâtiment $(\mathrm{n}=3207)$, par rapport aux branches Travaux Publics - Canalisation (TP Cana., $\mathrm{n}=1830$ ) et Génie Civil $(\mathrm{n}=711)$.

Pour les trois secteurs confondus, la structure d'âge de la population des salariés est vieillissante (cf. figure 1). On observe trois paliers d'importance croissante:

- Un premier où les moins de 30 ans représentent environ $16 \%$ de cette population;

- Un second, plus grand, où les 30-39 ans et les 40-49 ans représentent chacun $25 \%$;

- Un troisième où les 50 ans et plus représentent $34 \%$.

3. Cet effectif englobe les compagnons, les chefs d'équipe et les novices en formation; population étudiée ici. Il ne prend pas en compte la hiérarchie supérieure à $\mathrm{n}+1$ et les intérimaires dont les effectifs ne sont pas recensés au niveau du groupe. 


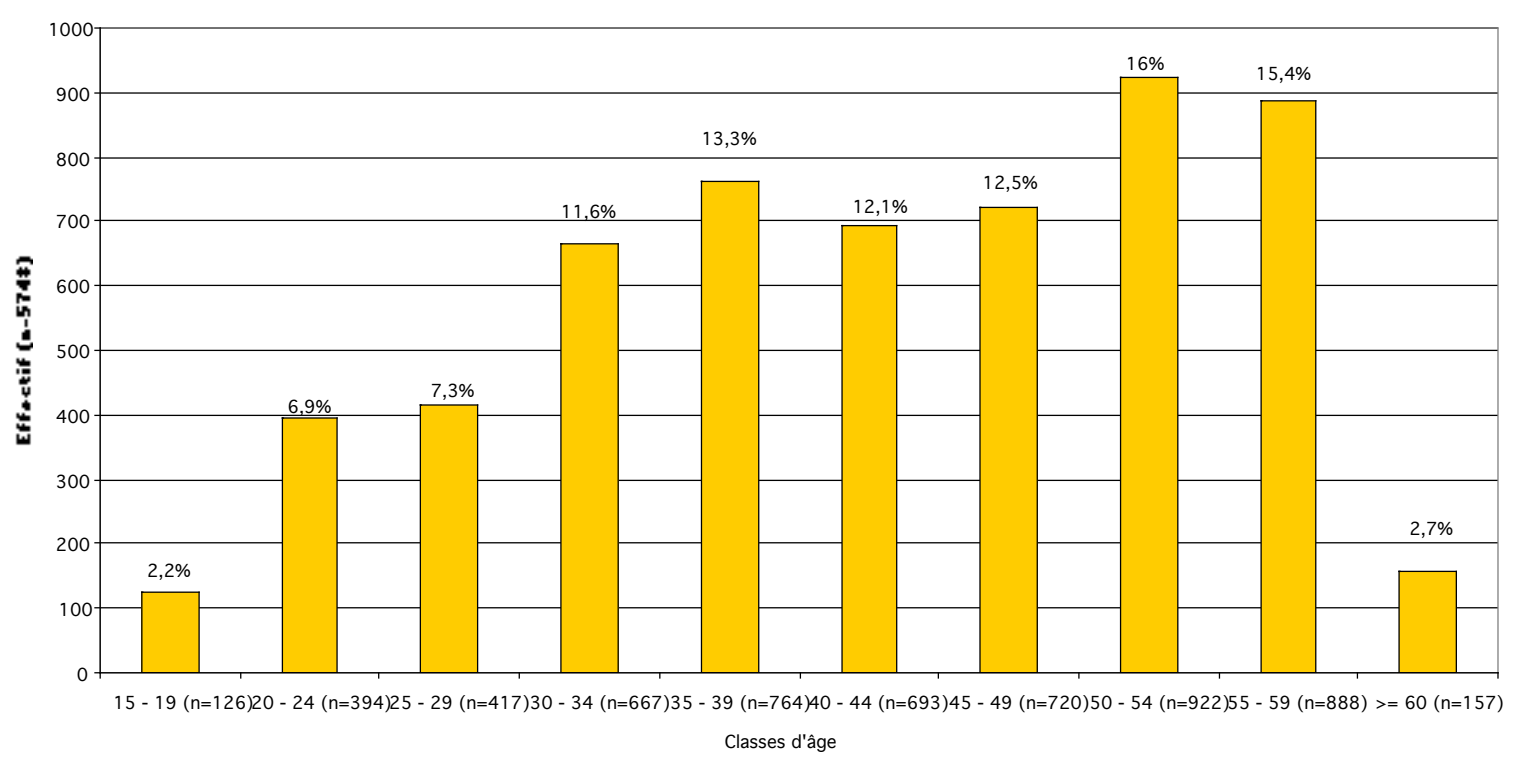

Figure 1: Effectifs des salariés de l'entreprise de Construction (Bâtiment, Travaux Publics - Canalisation, Génie Civil) selon l'âge pour l'année 2004 (n = 5748)

Figure 1: Staff count within construction company (Building, public works, canalization, civil engineering) by age for $2004(n=5748)$

Comparativement, cette tendance générale se retrouve dans chaque secteur d'activité, mais de manière plus ou moins contrastée. C'est dans le Bâtiment - secteur qui par ailleurs possède les effectifs les plus importants - que le vieillissement des ouvriers est le plus marqué, avec, en pourcentage, une plus faible proportion des moins de 25 ans contre une plus forte des 50 - 59 ans. Ainsi, d'un point de vue statistique, c'est dans ce secteur que les modifications de la structure d'âge vont être les plus importantes dans les années à venir, d'autant qu'il représente plus de la moitié de l'effectif total des salariés.

Les données RH montrent que le type de contrat le plus utilisé est le CDI, quel que soit l'âge. Il représente $95 \%$ des cas dans le Bâtiment et les TP Cana., un peu moins ( $88 \%$ ) dans le Génie Civil. Ce dernier utilise plus fréquemment le Contrat à Durée de Chantier que les autres secteurs: davantage concerné par des chantiers de longue durée mais itinérants, ce secteur a recours au CDC pour recruter de la main-d'œuvre locale, notamment pour les fonctions de manœuvre ou d'aide-coffreur requérant peu de qualifications.

Toutefois, la Direction Centrale des RH ne comptabilise pas les intérimaires (comme dans beaucoup d'autres entreprises). Or visites de chantiers et entretiens dévoilent un appel - non négligeable mais difficilement quantifiable - à l'intérim. Celui-ci concerne plutôt des jeunes, ce qui dans les faits tend d'une part à rajeunir la structure d'âge effective de la main-d'œuvre sur les chantiers et d'autre part à minorer la politique annoncée du contrat stable.

\section{2.- Des actions de formation qui ont des difficultés à séduire}

Face au vieillissement annoncé, l'entreprise tente de développer des actions de formation, en partenariat avec des organismes de formation, des sociétés d'intérim et des conseils régionaux. Globalement, sur une période de 18 mois entre 2003 et 2004, 166 personnes sont entrées dans un système de formation et 114 sont salariées de l'entreprise en novembre 2004. La répartition des formations et des embauches n'est toutefois pas équivalente d'un secteur à un autre: c'est le Génie Civil qui - sur cette même période - a formé $(n=96)$ et embauché $(n=56)$ le plus de personnes, alors que c'est le plus petit secteur en effectif. Le Bâtiment qui constitue $55 \%$ des effectifs n'a pas embauché beaucoup plus que les TP Canalisation, via le système de formation ( 32 embauches pour le bâtiment et 26 pour les TP Canalisation). 
Quel que soit le secteur, on note une déperdition importante de candidats à la formation entre la première étape, la réunion collective d'informations, et les deux premières semaines de formation, les pré-qualifications : 235 personnes se présentent dans le Génie Civil à la première étape et 96 restent en formation; 196 dans le Bâtiment pour 49 restantes; dans les TP Canalisation, la déperdition semble tout aussi importante car au moins 88 personnes se sont présentées à la réunion et au moins 21 sont restées en formation. En revanche, on note une stabilité des effectifs entre l'étape 2, (la préqualification) et l'étape 3 (le stage sur chantier): ceux qui s'engagent en formation la mènent, pour la plupart, jusqu'au bout. L'étape de l'embauche peut être beaucoup plus sélective: si certains chantiers - dans les 3 secteurs - embauchent la plupart de ceux qui ont fait leur stage, d'autres ne gardent environ que la moitié des candidats. Puis, ceux qui sont embauchés sont, pour la plupart, toujours dans l'entreprise dans l'année qui vient: les ruptures de contrat pendant la période d'essai et les démissions sont peu nombreuses quel que soit le secteur. Le taux faible de signatures de contrats de travail mais surtout le désengagement précoce d'un nombre non négligeable de prétendants (entre la réunion d'information et la formation) posent probablement la question de l'adéquation des attentes réciproques des futurs salariés et des employeurs. Mais nous voudrions aller au-delà des représentations en réfléchissant au poids des conditions de travail dans la phase d'accueil et de formation. À quelles réalités du travail ces nouveaux arrivants sont-ils confrontés?

\section{3.- Un apprentissage opportuniste}

\section{1.- Une diversité de statuts à l'entrée dans le secteur}

L'arrivée dans le milieu du BTP peut relever d'un choix initial anticipé ou d'une reconversion plus tardive. Pour une partie des salariés rencontrés, l'entrée dans ce secteur d'activité fait suite à une formation initiale suivie dans ce domaine (CAP tailleur de pierres, BEP métreur en bâtiments ou travaux publics, Bac Génie Civil, BTS Construction, etc.): «Je suis rentré en septembre, directement à la X, parce qu'ils sont venus me chercher à l'école pour m'embaucher. [...] Moi j'ai un CAP de poseur avec spécialisation de canalisation [...], mais c'est plutôt mon BEP de Travaux Publics qui m'a servi parce qu'on m'a demandé de faire de tout ». (Antoine 4,24 ans, Chef d'équipe, TP). Pour d'autres, l'arrivée dans ce métier correspond à une reconversion plus ou moins tardive après une formation ou une carrière dans des secteurs d'activités très divers (vente - commerce, comptabilité, coiffure, artisan boulanger). Ceci signifie que si la plupart des nouveaux sont plutôt jeunes, il n'est toutefois pas rare de rencontrer également des nouveaux de 35 ou 40 ans. Par ailleurs, dans de nombreux cas, comme nous l'avons vu précédemment, l'entrée dans ce secteur s'est faite dans la continuité d'une sensibilisation, voire d'une « éducation » familiale.

Du côté du statut des nouveaux, nous avons rencontré quatre formes différentes :

\section{Recruté en CDI}

Cinq personnes (sur 26) ont été recrutées en CDI : trois, anciennes dans l'entreprise, sont arrivées dans ce secteur par l'intermédiaire de connaissances familiales (père, frère ou beau-frère) et deux, plus jeunes, ont été recrutées à la sortie d'une école réputée, spécialisée dans le BTP, avec la proposition d'un encadrement par un tuteur pendant la première année afin de compléter leur formation. Toutefois, cette proposition ne s'est pas réalisée dans les faits et la prise en charge initiale a été quasi inexistante. Pour Benjamin ${ }^{5}:$ "j'ai signé avec la proposition d'avoir un tuteur pendant la première année. Arrivé là-bas, j'ai jamais eu de tuteur ». Pour Antoine ${ }^{6}$, c'est le conducteur de travaux qui avait été désigné officiellement comme son tuteur, mais il ne l'a jamais vu. Ils ont en fait débuté comme hommes-à-tout-faire, à l'identique des intérimaires: "c'est à nous de rentrer dans l'équipe

4. Tous les opérateurs ont été renommés par souci de garantir leur anonymat.

5. Benjamin:24 ans, CDI en sortie d'école BTP, Chef d'équipe, TP Cana.

6. Antoine : 25 ans, CDI en sortie d'école BTP, Chef d'équipe, TP Cana. 
et de faire nos preuves. Nous, on a un diplôme, donc c'est à nous de faire nos preuves » (Antoine). On comprend ici que le diplôme pourrait devenir en quelque sorte handicapant s'il était trop mis en avant.

\section{Stagiaire dans le cadre du parcours scolaire}

Nous avons rencontré très peu de personnes dans cette situation (3/26). Elles ont effectué des stages en entreprise dans le cadre de la préparation de CAP tailleur de pierre, BEP Construction ou BTS encadrement de chantier; elles ont été embauchées à l'issue de leur formation (diplôme validé ou non). Simon $^{7}$ a ainsi pu bénéficier d'une fonction d'encadrement, puisqu'il avait un tuteur administratif et un tuteur de terrain, en l'occurrence le chef d'équipe. À la différence des autres situations rencontrées, il est surnuméraire dans la plupart des équipes qu'il rencontrera, c'est-à-dire qu'il n'est pas comptabilisé dans les ratios de production, sa tâche première étant alors officiellement de se former. Un mode de prise en charge avait été prévu et il a ainsi changé plusieurs fois d'équipe avec l'objectif de se faire une idée du fonctionnement d'un chantier. Il insiste beaucoup sur l'influence d'être surnuméraire ou non pendant cette période d'apprentissage: «Sur le chantier béton, j'allais voir le chef de chantier, il me montrait les plans et je passais un peu partout pour voir ce que les gars faisaient. Sur les chantiers d'assainissement, je passais ma journée au remblai. Quand on est au rendement, on ne peut pas se permettre de quitter son poste pour aller demander quelque chose ».

\section{Intérimaire}

Ce n'est pas un statut de formation à part entière, mais c'est pourtant le statut majoritairement rencontré pour se former (11/26), particulièrement dans le bâtiment et les travaux publics de canalisation: on commence dans la profession comme intérimaire en souhaitant se faire embaucher en CDI. Ceux que nous avons rencontrés étaient d'anciens intérimaires qui « ont réussi » à se faire embaucher, après une période d'intérim plus ou moins longue, 5 ou 6 ans parfois. La formation n'y est pas officielle et la prise en charge des nouveaux est donc succincte, voire inexistante. Si le chef d'équipe souhaite la présence d'un intérimaire, c'est parce qu'il a besoin d'une main-d'œuvre supplémentaire qui participe d'emblée à la production. De ce fait, on attribue à l'intérimaire des tâches qui ne nécessiteraient apparemment aucune formation: «on tient la pelle » et cela peut durer longtemps. Nous avons rencontré toutefois deux situations où les intérimaires ont été pris en charge explicitement par une personne de l'équipe pour être formés: dans un cas, il s'agissait d'une personne sur le point de partir à la retraite et qui a formé l'intérimaire avec l'objectif qu'il assure son remplacement; dans l'autre cas, il s'agissait d'un chef d'équipe, seul, qui recrutait une première personne et c'est dans le cadre de ce binôme que l'intérimaire a été formé. On voit ici que l'objectif de formation est clairement affiché quand un besoin immédiat de recrutement dans l'équipe et pour le long terme apparaît explicitement.

\section{Recruté en Contrat à durée de Chantier}

Compte tenu du caractère itinérant de certains chantiers, particulièrement dans le génie civil, l'entreprise recrute du personnel (7/26) dans les régions où se situent ces chantiers sur la base de contrat dont la durée équivaut à la durée du chantier (1 à 3 ans environ). Là encore, ce statut ne préjuge en rien du mode d'accueil des nouveaux: d'une prise en charge formalisée à une prise en charge quasi inexistante.

Les entretiens réalisés laissent entendre une prise en charge homogène, calée sur celle de l'intérimaire, même quand un dispositif de tutorat est prévu. Ce mode d'accueil des nouveaux reproduit celui évoqué par de plus anciens, même trentenaires, où il s'agissait avant tout de faire ses preuves. Ainsi, les débuts de chefs de chantier expérimentés d'aujourd'hui sont marqués par le statut de «mousse » ou de manœuvre, quelle que soit la forme du contrat, intérimaire, en contrat de chantier ou en ap-

7. Simon: 24 ans, stagiaire BTP, chef d'équipe, TP Cana. 
prentissage: « Je suis rentré manœuvre. Ça a dû durer facilement 6 mois. » $\left(\right.$ Mikaël $\left.{ }^{8}\right)$. « On est un petit peu le mousse du chantier. On est l'aide coffreur, l'aide-maçon, on est le mancuvre. On passe partout, on assure la propreté du chantier, on donne un coup de karcher sur les banches, ce qui se fait de moins en moins... C'était une transition, ça a duré 6 mois ou 1 an. » (Franck $\left.{ }^{9}\right)$.

Tous considèrent que cette première expérience des chantiers est éprouvante physiquement et moralement: "Mon premier chantier, je l'ai très mal ressenti. J'en étais dégoûté, j'ai failli quitter le chantier d'ailleurs. [...] Suite à différentes altercations avec des anciens, je me suis dit «tu vas pas pouvoir continuer comme ça». [...] Au départ physiquement, moi j'étais un peu casse-cou puisque je venais des commandos de l'armée, mais physiquement c'était fatigant. Il y avait une grosse partie morale parce qu'on se sentait dégradé. Quand on a passé une journée à remplir une benne de sable avec une pelle, vous rentrez le soir, vous êtes fatigué parce que ça use une pelle, mais d'un autre côté vous êtes encore plus fatigué dans votre tête. Moi, je ne suis pas ingénieur, mais je me voyais mal toute ma vie avec une pelle à gratter. C'était dégradant. Ça psychologiquement, ça m'a quand même atteint. Je me suis senti régresser dans la vie. » (Mikaël).

\section{2.- Une homogénéité des formes d'accueil et d'apprentissage}

L'analyse rétrospective des parcours professionnels des opérateurs montre une relative homogénéité des modalités d'accueil et de formation, quel que soit leur statut à l'arrivée.

\section{Comment ont-ils appris?}

Pour tous les opérateurs rencontrés, l'apprentissage peut être qualifié « d'opportuniste », dans le sens où ils tentent de tirer le meilleur parti des circonstances; les nouveaux doivent eux-mêmes prendre en charge leur apprentissage et leur début de parcours professionnel. Ils ont tous débuté manœuvres et ont dû faire leurs preuves avec une vision approximative, voire nulle, de leurs possibilités d'évolution. Ils ont ainsi dû trouver seuls l'utilité de commencer manœuvre et faire eux-mêmes le lien entre les exigences requises, par leurs pairs et la hiérarchie, relatives aux comportements (i.e. être courageux, volontaire, s'intéresser, etc.) et le contenu du travail. Ils ont dû s'imposer en prouvant qu'ils étaient en mesure de faire le travail de l'autre: en arrivant plus tôt pour faire le travail, en profitant des pauses ou des absences de collègues, en prenant les outils des mains des anciens, voire en passant outre les décisions hiérarchiques: "Le projeteur s'est cassé la main et c'est moi qui ai repris [...] J'ai fini le chantier et c'est comme ça que je suis resté dans l'entreprise » $\left(\mathrm{Jean}^{10}\right)$. "C'est en posant les questions et en prenant les outils des anciens. Il faut les leur prendre des mains, parce que si on n'insiste pas, ils ne les prêtent pas [...] Il faut les bousculer "laisse-moi faire », sinon on passe la journée à les regarder et c'est tout " (Nicolas $\left.{ }^{11}\right)$. "Chaque machine que je voyais, Tac, je montais dessus. Alors des fois je me faisais engueuler, le chef il venait: descend de là, t'as rien à faire dessus! Mais moi le lendemain, je remontais dessus et le mec me réengueulait. Et puis il s'est trouvé des jours où le conducteur d'engins n'était pas là. Et le chef: bé tiens, Mikaël, t'aime bien les machines, monte. Et au bout d'un an, je me suis retrouvé sur une machine, sur une fraise. En dessous du chef d'équipe, c'était le rôle principal ». (Mikaël).

L'affût des opportunités de montrer ses capacités s'est accompagné d'une démarche proactive pour acquérir de nouvelles connaissances, se traduisant par la combinaison de plusieurs stratégies:

- Regarder travailler les plus expérimentés.

Regarder de près : être affecté aide-poseur permet de voir de près travailler le poseur - « Le but

d'un bon manœuvre, c'est que le poseur en question, il ait le moins possible de choses à faire en

8. Mikaël : 33 ans, CDI, Chef d'équipe, Bâtiment.

9. Franck: 36 ans, stagiaire, Chef de Chantier, Bâtiment.

10. Jean: 31 ans, intérimaire, compagnon, TP Cana.

11. Nicolas : 34 ans, intérimaire, chef d'équipe, TP Cana. 
dehors de la pose; ça fait beaucoup de préparation de matériels » (Benjamin).

Regarder de loin: être affecté au remblai ou à la conduite de la pelleteuse permet d'avoir une vue d'ensemble du chantier.

Regarder le plan: c'est un signe de confiance du chef d'équipe; cela permet de comprendre le chantier dans sa globalité et d'anticiper son travail sur la journée.

- Poser des questions: «Ceux qui m’ont appris le métier: des soudeurs, des tuyauteurs, parce que ça m'intéressait. Je posais des questions, j'allais voir comment ils travaillaient. Dès que j'avais 5 minutes, j'allais voir le tuyauteur et je parlais avec lui » (Nicolas); « On nous explique si on demande » (Simon); « J'ai appris en regardant. Les explications viennent si on s'entend bien avec la personne » $\left(\right.$ Christophe $\left.^{12}\right)$.

- Faire le tri, i.e. adapter ce que les autres ont montré à ses propres caractéristiques - ce qui implique de pouvoir prendre du recul sur sa propre pratique, autrement dit d'avoir une pratique réflexive. «Après, c'est propre à chacun de faire le tri, de telle et telle méthode. Je crois que ça fait partie des ficelles du métier » (Franck).

Le statut du nouveau n'a que peu d'incidence sur ses débuts: celui qui ne possède aucune expérience et pas de diplôme commence manœuvre car il ne peut réaliser une tâche nécessitant des connaissances techniques spécifiques, et celui qui a un diplôme ne doit pas être favorisé par rapport aux autres et doit « faire ses preuves » comme les autres. En revanche, ce statut d'homme-à-tout-faire peut durer moins longtemps que pour les intérimaires et ils ont pu rapidement évoluer vers d'autres tâches: entre 6 et 12 mois après, ils sont aujourd'hui statutairement aide-poseurs, et dans les faits, poseurs et épaulant le chef d'équipe. Ainsi, si les débuts sont similaires à ceux des intérimaires, les opportunités de carrière se développent plus rapidement.

\section{Comment accueillent-ils et transmettent-ils?}

Alors que les nouveaux se focalisent sur l'apprentissage de la dimension technique du métier et sur la compréhension de l'organisation du chantier, les plus anciens leur demandent d'abord de faire preuve de leur vaillance, de leur courage, d'avoir du respect et de s'intéresser à la vie de chantier. Plusieurs extraits d'entretiens en montrent l'importance: "les intérimaires sont plus ou moins vaillants ou prêts à recevoir des ordres. On essaie de voir les intérimaires qui sont capables de faire quelque chose. Au bout de 4, 5, 6 mois, on fait du tri, voir s'ils sont courageux " $\left(\right.$ Gérard $\left.^{13}\right)$; "Il faut bien commencer. Moi, j'ai commencé terrassier, faire des sondages. J'en voulais, je faisais mon travail, je le faisais bien et j'avais envie. Ça, ils le voient tout de suite quand un jeune a envie. On est là à lui expliquer, lui faire voir. Après, quand on tourne le dos, il a plus envie. Il commence à s'appuyer sur sa pelle. Et après quelque temps, ils s'en vont » (Nicolas). Les premières tâches confiées aux nouveaux ne sont pas considérées par les anciens comme nécessitant des compétences spécifiques: tenir la pelle ne s'apprend pas.

Les exigences requises par les « anciens » peuvent être ambivalentes pour les nouveaux à qui on demande de donner d'eux-mêmes, de montrer leur intérêt (en posant par exemple des questions), tout en exécutant les ordres sans les discuter. Par ailleurs, les qualités requises - vaillant, courageux, respectueux - sont des qualités a priori déconnectées du contexte du travail; les « anciens » font comme si être courageux était une qualité naturelle, innée, indépendante du travail et des conditions dans lesquelles il est réalisé. Ce sont par ailleurs des qualités qui doivent être tenaces, dans la mesure où la période de mise à l'épreuve peut durer plusieurs mois, voire années, dans un contexte où le nouveau a peu d'informations sur ce qui est susceptible de lui arriver, tout en percevant qu'il doit prendre son parcours en main.

Du côté des « anciens », leurs propos laissent entendre que leur attitude s'est construite aussi sur les échecs qu'ils ont pu rencontrer, particulièrement avec des intérimaires pour lesquels ils se sont investis et qui ne sont pas restés. Quand ils évoquent leurs propres débuts il y a 15 ou 20 ans, ils notent

12. Christophe: 38 ans, CDI, compagnon, TP Cana.

13. Gérard: 44 ans, intérimaire, chef d'équipe, TP Cana. 
que c'était alors les plus anciens de l'époque qui s'inscrivaient dans une démarche proactive: «les compagnons disaient comment il fallait faire et pourquoi on devait le faire comme ça. Il faut expliquer plusieurs fois. [...] En regardant et en refaisant petit à petit, on refaisait, ils regardaient si on faisait pas de bêtises » (Gérard). La stabilité de l'équipe et les contraintes de temps sont également évoquées comme un facteur important pour créer un collectif de travail et transmettre dans de bonnes conditions: "Expliquer aux nouveaux? Ça dépend si on est en retard ou pas. Et on ne sait pas à l'avance combien de temps les intérimaires vont rester »(Jean).

La gestion et la prévention des risques sont prises officiellement en charge par les chefs de chantiers lorsqu'ils encadrent des stagiaires ou des intérimaires. Les trois chefs de chantier rencontrés considèrent qu'ils n'ont rien à apprendre aux intérimaires, y compris aux novices dans le BTP, à l'exception des consignes de sécurité et des pièges du chantier. Lorsqu'ils suivent des stagiaires en formation, elle consiste à les faire tourner sur plusieurs tâches pour identifier ceux qui pourront être polyvalents. Ils sont également soucieux de leur apprendre la discipline dans le travail, comme arriver à l'heure, prendre ses pauses à l'heure dite. La transmission des connaissances et savoir-faire n'est en fait abordée que très rarement. Les chefs de chantier délèguent ces dimensions aux compagnons.

Ces entretiens, agrémentés de visites de chantier, ne prétendent aucunement à l'exhaustivité. Il ne s'agit donc pas de tirer des généralités, mais plutôt d'évoquer des tendances rencontrées dans les différentes branches d'activité de l'entreprise. Nous voudrions ainsi pointer plusieurs éléments:

- La question du statut de nouveau semble avoir peu d'incidence sur le début de son parcours professionnel et le contenu de son apprentissage. On demande aux nouveaux d'exécuter des tâches de manœuvre ou périphériques au chantier, peu qualifiées mais directement productives. Pour ceux qui ont eu l'occasion d'accueillir des nouveaux - et parmi eux des trentenaires - il ne s'agit pas tant dans cette étape de transmettre des savoirs et des gestes professionnels que d'apprécier et de tester l'implication des nouveaux, à l'image de leurs propres débuts.

- La mise en place d'un dispositif d'accompagnement et de formation de nouveaux est toutefois évoquée à plusieurs reprises. Mais elle semble alors souvent rattachée à un enjeu de court terme faisant apparaître comme essentiel d'avoir un opérateur formé dans de brefs délais, ou à des motivations d'ordre individuel d'un ancien pour prendre en charge un nouveau. Même la définition contractuelle d'un encadrement n'est pas une garantie qu'elle se réalise;

- La transmission des savoirs et savoir-faire professionnels n'étant ni prise en charge par les anciens, ni par l'encadrement, ou à la marge, les nouveaux se trouvent donc être à l'initiative de cet apprentissage, montrant par là même leur intérêt à apprendre le métier. Cet apprentissage peut s'avérer plus ou moins douloureux, tant physiquement que psychiquement, particulièrement quand les perspectives d'emploi et la durée de cette phase restent floues.

Nous voudrions maintenant examiner comment se joue actuellement la transmission dans une situation où sont posés d'emblée la formation et l'accueil de nouveaux, dans un dispositif d'envergure associant l'entreprise à un organisme public de formation. La transmission entre nouveaux et anciens en est-elle modifiée? Quelle place est alors accordée à l'apprentissage des savoirs et savoir-faire professionnels?

\section{4.- L'apprentissage le métier de « coffreur-bancheur»}

\section{1.- Le dispositif de formation}

L'entreprise, en partenariat avec des organismes sociaux et de recherche d'emploi régionaux et un organisme de formation pour adultes, a mis en place une formation de « coffreur-bancheur », transversale à ses trois branches d'activité, le bâtiment, les travaux publics de canalisation et le génie civil. Ce dispositif prévoit une formation dans un centre d'apprentissage, puis sur un chantier, avec à la clef une possibilité d'embauche après un dernier entretien d'évaluation. Le dispositif évoqué ici se 
déroule en région Centre avec une formation de terrain sur un chantier de génie civil devant réaliser trente ponts sur un tronçon d'autoroute. La formation proposée s'est déroulée en plusieurs étapes.

\section{- Une réunion d'information}

Une journée d'information collective est organisée après la diffusion d'offres d'emploi d'aide-coffreur dans la région et a réuni 85 personnes. Le DRH du site, ainsi que des conducteurs de travaux du chantier, présentent l'entreprise et les possibilités d'évolution de carrière du fait, notamment, des nombreux départs à la retraite à venir. La formation proposée permet d'obtenir un statut d'aide-coffreur qui, avec un ou deux ans de pratique, évolue vers le statut de coffreur. À l'issue de cette présentation, les personnes intéressées, au nombre de 70, ont passé un entretien individuel portant sur leur parcours scolaire et leurs motivations. Les autres partent.

\section{- Une pré-sélection en stage de remise à niveau générale}

Cette formation, dispensée par l'organisme de formation, porte essentiellement sur les mathématiques et le français. D'une durée de deux semaines, elle s'adresse à des groupes d'une quinzaine de personnes de niveaux très variés, certains sachant à peine lire et d'autres ayant le niveau d'un bac professionnel. Seules 34 personnes achèvent cette étape suivante.

\section{- La formation de coffreur-bancheur}

Cette formation, également prise en charge par l'organisme de formation, dure cinq semaines. Elle comporte des parties théorique et pratique sur les coffrages, les niveaux, les armatures, la résistance des matériaux, les étaiements et le béton. Deux groupes de 17 stagiaires sont constitués. Le premier effectue sa formation en mai; le second en juillet. Les 34 stagiaires terminent cette étape.

\section{- Le stage sur le chantier}

Durant trois semaines les stagiaires sont suivis par un formateur du centre qui leur rend régulièrement visite sur le chantier. C'est au cours de cette étape que des analyses de l'activité ont été réalisées. Quatre stagiaires du second groupe abandonnent avant la fin du stage.

\section{- La réunion pour le recrutement final}

L'ensemble des acteurs de cette formation (DRH, conducteurs de travaux, directeur de chantier, chefs de chantier, organismes sociaux et de recherche d'emploi, organisme de formation) se réunit pour décider des stagiaires acceptés en Contrat à Durée de Chantier. Les stagiaires sont ensuite informés individuellement. Dans le premier groupe, 10 sur 17 se sont vus proposer un CDC. Dans le second groupe, 8 sur 13 sont embauchés en CDC, mais 2 abandonneront pendant la période d'essai.

Au final, sur 70 personnes volontaires à l'issue de la première réunion d'information, 16 sont en CDC quelques semaines plus tard. Comment expliquer une telle déperdition de candidats, alors même que la formation est qualifiante et peut déboucher sur un emploi?

\subsection{Les modalités de transmission du métier}

Nous avons réalisé des analyses de l'activité à partir d'enregistrements vidéo de 10 opérateurs d'âges et d'anciennetés différents, dont trois sont issus de la formation « coffreur-bancheur ». Sur la base de ces analyses, certaines actions, centrales dans la pratique, (le talochage, le serrage d'un écrou papillon, le coffrage d'un angle) ont été retenues pour mettre en évidence les conditions d'élaboration de certains de ces savoir-faire. Elles ont été reprises dans des montages vidéo de quelques minutes et visionnées au cours de seize entretiens individuels en autoconfrontation (Chassaing, 2006). Certaines d'entre elles reprennent des situations de transmission, c'est-à-dire des échanges entre nouveau/ancien en co-présence. Globalement, nous retrouvons le caractère opportuniste de l'apprentissage, tant du côté de ceux qui transmettent que du côté de ceux qui apprennent. 


\section{- Qui enseigne?}

Différents acteurs au sein de l'équipe jouent un rôle dans l'apprentissage du métier. La plupart du temps, c'est le chef d'équipe ou un coffreur plus ancien qui transmet. Toutefois, les nouveaux apprennent majoritairement seuls par essai/erreur. Les échanges ont alors lieu quand le nouveau est mis en échec. La transmission a aussi lieu en fonction de la tâche effectuée: si la tâche comporte un enjeu de sécurité ou de qualité, un « tuteur » est présent avec le nouveau pour réaliser le travail et pour le contrôler. Il n'existe pas ici de réelle systématisation concernant l'encadrement. Les chefs d'équipes et certains coffreurs semblent garder un œil sur les nouveaux et interviennent quand ils le jugent nécessaire, en fonction des enjeux de sécurité et de production.

\section{- La transmission de savoirs gestuels au détour du travail des anciens}

Quand la transmission s'opère, les analyses permettent de souligner l'importance du travail des autres, des échanges avec des travailleurs expérimentés pour l'apprentissage de savoir-faire gestuels et le développement de pratiques. La pratique des autres, comme source de construction des actions analysées, se manifeste selon différentes modalités. Nous nous sommes inspirées des stratégies présentées par Cloutier, Lefebvre, Ledoux, Chatigny et St-Jacques (2002), pour analyser nos situations de transmission:

\section{- Laisser faire en regardant et en donnant des instructions}

Un ancien est en hauteur sur le coffrage et le nouveau est en bas; l'ancien lui dit quel écrou papillon serrer et il l'observe taper sur le papillon avec le marteau, puis lui dit «stop arrête ça suffit ». L'ancien lui explique que lorsque l'action de frappe du marteau sur l'écrou papillon émet un son aigu, cela signifie que l'écrou est suffisamment serré. À cette occasion, l'ancien transmet un principe de base essentiel au geste du serrage des écrous papillons dans n'importe quelle situation qui est «écouter le son du papillon ». Le recours aux ressources sensorielles pour guider le geste semble être un moyen qui se développe avec l'ancienneté et la connaissance de la tâche. Les moins anciens n'évoquent pas ce principe de base en lien avec des composantes sensorielles auditives.

\section{- Montrer en expliquant puis laisser faire et regarder}

Un nouveau est en train de brancher une « soufflette » pour nettoyer du béton; il éprouve des difficultés à relier le tuyau de la « soufflette » à celui du groupe électrogène; un expérimenté le voit et décide de lui montrer en lui expliquant comment faire; il demande ensuite au nouveau de refaire le geste devant lui.

- Donner un conseil quand le nouveau est mis en échec

Les conseils des anciens, de collègues, et donc leurs interventions pour aider un apprenant en difficultés, sont une source de développement:

\footnotetext{
«Ergonome: et au début les premiers trucs qui n'allaient pas pour talocher c'était quoi? Arnaud (25ans, nouveau) : c'est euh... ben je prenais mal la taloche.

E: tu la prenais comment?

Arnaud: ben je la prenais... bon y'a une poignée comme celle-là (sur la vidéo), mais Daniel (55 ans, ancien) il me dit tu fais des cercles comme ça, et moi je n'y arrivais pas, je creusais le béton... Daniel il m'a montré comment il fallait faire pour la prendre sans trop appuyer dans certains endroits, puis après j'y suis arrivé ».
}

\section{- Montrer en expliquant}

Un ancien montre à un nouveau comment planter une « carotte » (tige de fer) pour fixer un étai qui part du sol vers le panneau et assure ainsi son maintien. Il lui montre en lui expliquant: commencer par faire un trou dans la terre avec le marteau (trou d'environ $3 \mathrm{ou} 4 \mathrm{~cm}$ de profondeur) de la largeur de la base de l'étai, placer la base de l'étai puis enfoncer la carotte à coup de marteau dans le trou de la base de l'étai, enfin relier la carotte à l'étai avec du fil de fer doublé. 
- Guider et faire ensemble

L'apprentissage se poursuit au-delà de la période de formation proprement dite. "Poser un fer » à deux contre un coffrage a été l'occasion d'observer une transmission d'un savoir-faire entre un opérateur ancien et un autre moins expérimenté qui a découvert une autre façon de faire, plus efficiente, que celle à laquelle il songeait.
«E: d'accord... le fait d'être à deux pour faire ça c'est important?
François (23 ans, nouveau) : c'est bien, ah oui c'est important, surtout là avec les anciens comme ça c'est bien. On apprend, on apprend beaucoup. Ca gueule beaucoup mais on apprend beaucoup aussi.
E: oui, même dans le cas de poser le fer là par exemple?
François : c'est des trucs... des fois des trucs tout simples (...) moi à l'origine... déjà à l'origine je voulais le prendre à la main et le passer derrière, donc droit...(Passer le fer horizontalement par- dessous la passerelle), et le prendre à deux mais il m'a dit (il l'imite) "non, non ça sert à rien de forcer! On va le passer par en haut, en biais et ça va passer tout seul».
E : d'accord, d'accord... donc toi dans cette situation là toi tu aurais choisi de le passer...
François :...oui, on aurait peut-être forcé un peu plus mais on aurait pu le mettre droit tout
simplement, mais bon... c'est vrai que c'était pas facile d'accès parce qu'il fallait passer dessous, après on a le terrain il est comme ça en bas (mime avec sa main la pente du talus)...
E: oui, oui il y'avait le talus là...
François : ...mais ça aurait pu se faire aussi (...) mais il (l'ancien) a dit y'a la grue donc on va se servir de la grue, c'est vrai qu'il avait raison parce que... quand on peut éviter de forcer c'est bien aussi.»

- Laisser faire seul sans instruction, puis contrôler le résultat

Cette stratégie est surtout utilisée dans des tâches de finition où les nouveaux sont souvent seuls; le chef donne des directives, laisse faire le nouveau puis revient pour contrôler le travail. Il arrive que le nouveau qui fait de la finition sur des arêtes (ôter le béton au burin ou le poncer) réalise des actions non nécessaires; ce n'est qu'une fois que le chef vient contrôler le travail, qu'il lui dit que ce n'était pas la peine d'ôter le béton sur cette partie car elle n'est pas visible.

\section{- Tester}

Certains testent les nouveaux en leur donnant une tâche à réaliser sans but, comme creuser un trou. Le chef attend du nouveau qu'il se demande pourquoi il doit creuser un trou, il veut que le nouveau sache ce qu'il fait. Il le laisse creuser tant qu'il ne demande pas pourquoi. Dans une autre situation, un nouveau doit planter un clou dans une posture contraignante: il doit le planter pour fixer une planche de bois contre un panneau et il n'arrive pas à taper suffisamment fort compte tenu de la position dans laquelle il est. De ce fait, le clou se tord sans être bien enfoncé: il l'enlève et recommence avec un autre. Un ancien au dessus lui dit « je te le dis pas mais tu as $1 \mathrm{~cm}$ d'écart au moins »; l'ancien lui signifie par là que l'écart entre la planche et le panneau est trop grand pour qu'il y arrive. Puis l'ancien repart à sa tâche et le jeune reste en échec. Le chef d'équipe arrive alors et le regarde faire sans rien dire; le nouveau continue de tordre les clous. Le chef le réprimande parce qu'il ne porte pas le casque et le nouveau répond qu'il le gêne pour planter ce clou. Le chef lui fait une remarque " $t u$ mets du temps pour planter un clou! », il le regarde puis lui dit d'arrêter et lui dit d'aller placer des garde-corps. Le chef prend le relais et plante le clou. Ici le nouveau est mis à l'épreuve différemment, le chef ne lui explique pas comment faire et le renvoie à une autre tâche.

La plupart des apprentissages se font seuls, par essai/erreur. Quand ils sont supervisés par un ancien, ils se réalisent souvent par l'observation du nouveau des façons de faire de l'ancien. Il n'est toutefois pas certain que ce mode d'apprentissage suffise pour apprendre et faire évoluer sa pratique, dans la mesure où il ne permet pas d'accéder aux principes de base qui permettent de guider un geste. Nos observations et nos analyses menées sur l'action de coffrage d'un angle le confirment:

$$
\begin{aligned}
& \text { «E: il faut le savoir au début qu'on peut contrôler «l'à fleur » avec la main comme ça, c'est pas } \\
& \text { instinctif? }
\end{aligned}
$$


Denis (33 ans) : c'est en travaillant avec les anciens ça, on voit qu'ils mettent la main, donc au départ on sait pas pourquoi vu qu'on connaît pas et après... c'est vrai au début on peut se dire ils tiennent le contreplaqué mais non en fin de compte il tient, mais il met bien en même temps «l'à fleur ». Donc ça au départ on le voit faire mais on le fait pas forcément. Puis après, quand on s'aperçoit que le coffrage, il est pas bien, on dit «merde »! En fin de compte... bon on met la main puis on essaie de s'appliquer bien pour sentir les sensations avec la main de façon que ce soit bien mis.».

On repère bien ici la nécessité de remonter au principe pour apprendre. C'est ce qui est de l'ordre du sensoriel dans le geste qui semble faire l'objet d'un apprentissage plus approfondi, et qui caractérise l'opérateur expérimenté dans cette action du coffrage d'angle.

\section{3.- Des conditions d'encadrement et de mise en ouvre peu favorables à l'apprentissage}

À ce contexte d'apprentissage et de transmission s'ajoutent d'autres éléments qui ont joué en défaveur des jeunes en formation.

\section{- Un déficit d'anciens dans les équipes}

Certaines équipes du chantier accueillaient davantage de stagiaires que d'anciens. L'une d'entre elles était même constituée uniquement de stagiaires. Ces derniers sont alors encore beaucoup plus dans l'improvisation, en fonctionnant par essai/erreur. Sans nier les vertus possibles de ce mode d'apprentissage, il peut nécessiter d'une part plus de temps et, d'autre part, placer les stagiaires dans des situations à risque. Ce déficit d'anciens est dû, pour partie, à un élément conjoncturel, la période de la formation. L'accueil sur le chantier s'est fait en été, au moment où de nombreux salariés étaient partis en vacances. De fait, beaucoup de stagiaires ne se sont pas trouvés en situation surnuméraire, mais dans l'effectif de production. Faiblement expérimentés, ils étaient souvent considérés comme manœuvre, affectés à des tâches périphériques, telles que le nettoyage, le transport d'outils pour les anciens, le placement des garde-corps. Si ces tâches peuvent faire partie du métier, ce contexte a créé un décalage de représentation chez les stagiaires, entre formation reçue et réalité du chantier. Par ailleurs, ce motif conjoncturel s'associe à un autre, plus structurel. Les évolutions technologiques du métier et les nombreux départs à la retraite passés et à venir conduisent à une disparition des savoir-faire utilisés dans le coffrage traditionnel. Cette situation conduit à de fortes inquiétudes de la hiérarchie et des anciens eux-mêmes qui font un lien direct avec la croissance des défauts sur les ouvrages. Alors que la formation est conçue pour pallier cette situation, peu de stagiaires ont été désignés pour apprendre cette part du métier. Enfin, les contraintes de temps, dans ce contexte de déficit d'anciens et d'expérience, s'en sont trouvées renforcées au détriment de la formation sur le terrain.

\section{- Des tensions entre tuteurs}

Sur le chantier, beaucoup - tant du côté de l'encadrement que des anciens - se sont plaint que la période d'apprentissage au centre de formation était insuffisante. Plusieurs motifs ont été évoqués: une formation décontextualisée, du fait de sa transversalité aux trois branches d'activité; une formation partielle qui a plus mis l'accent sur des techniques de coffrage principalement utilisées dans le bâtiment; un public trop hétérogène où certains ont appris beaucoup et d'autres presque rien. Ces critiques ont créé des tensions entre les tuteurs du centre de formation et les tuteurs de chantier et ont participé à désorienter un peu plus les nouveaux.

\section{- Des tensions entre anciens et nouveaux}

Elles relèvent de divergences de représentations du métier, et plus généralement du travail, entre les nouveaux, pour la plupart jeunes, et les anciens. Celles-ci ont conduit à des conflits plus ou moins latents et à des incompréhensions mutuelles. Apprendre le métier, pour les anciens, implique d'éprouver la pénibilité du travail, des blessures physiques, d'obéir aux ordres sans discuter: apprendre le métier, c'est apprendre à endurer. Cette conception du travail et les modes de relations profession- 
nelles qu'elle engendre heurtent de nombreux jeunes qui, par ailleurs, ne conçoivent pas le travail comme devant « faire mal ». Ces tensions ont été renforcées par des inégalités salariales, ressenties par les plus anciens dont le niveau de salaire était quasi identique à celui des nouveaux. Ces conflits ont une incidence sur les opportunités d'apprentissage pour les plus jeunes, dans la mesure où c'est le jugement des anciens sur leur « vaillance » et leur « volonté » qui décidera en partie des conditions et du contenu de l'apprentissage.

Ainsi, à l'identique des situations d'apprentissage évoquées dans le Bâtiment et les TP Cana., ce dispositif produit lui aussi un apprentissage opportuniste du geste professionnel. Ce dispositif de formation le considère comme une somme d'habiletés techniques simples à acquérir, alors que le geste professionnel renvoie à l'articulation complexe de plusieurs dimensions, physiques, sensorielles, cognitives et même sociales puisqu'il s'inscrit et se construit dans des collectifs et des règles de métier (Clot, 1999 ; Bourgeois, \& Hubault, 2005). La formation de cinq semaines au centre associée à trois semaines de stage sur le terrain est considérée comme suffisante pour régler l'apprentissage du coffrage. Ce sont alors des dimensions comportementales qui sont mises en avant pour faire la différence entre deux candidats au Contrat à Durée de Chantier. Lors de la dernière phase de recrutement réunissant les acteurs de ce dispositif, les critères qui sont entrés en ligne de compte faisaient référence à l'esprit d'initiative, le volontariat, la vaillance, la compréhension des consignes, l'assiduité etc. et ne relevaient aucunement des savoir-faire développés.

\section{5.- Fidéliser par l'accueil et la formation?}

\section{1.- Mettre la fidélisation au centre de l'organisation du travail}

La question de la fidélisation des nouveaux s'avère complexe et multidimensionnelle. Portée au niveau d'une direction centrale des ressources humaines du groupe qui a pris la mesure de l'impact du vieillissement de sa population, elle se traduit - comme dans la plupart des cas (Pichault, \& Nizet, 2000) - par la mise en place de dispositifs d'accueil et de formation. Mais elle peut alors se heurter - comme c'est le cas ici - à une politique plus locale de gestion du personnel prise dans des contraintes économiques de court terme et débouchant sur l'utilisation de l'intérim ou du contrat à durée de chantier. La superposition de ces deux « outils GRH» brouille certainement les repères à tous les niveaux. Berthet et Cru (2003) constatent que «l'hétérogénéité et les pertes de repère sont deux tendances lourdes de la prescription» (p. 89). Elles conduisent à un sentiment que tout échappe, qu'il n'y a plus de prise sur le travail et les relations au travail. Lorsque ces actions d'accueil échouent, les interprétations des problèmes renvoient aux caractéristiques psychologiques et aux comportements sociaux des protagonistes: absence d'attitude collaborative, manque de courage, de respect de la hiérarchie... Les échecs sont personnalisés : c'est alors Paul qui n'écoute pas ce qu'on lui dit. Les différences d'âges sont naturalisées: d'une part, les jeunes ne voudraient pas apprendre, ne s'investiraient pas suffisamment dans le travail; d'autre part, les âgés ne voudraient pas transmettre. Les problèmes sont ainsi externalisés, poussés hors des murs de l'entreprise, et l'on invoque l'évolution de la Société et des valeurs en général sans connaître vraiment ce à quoi elles faisaient référence auparavant (Rousselet, 1987). Dans tous les cas, les solutions ne sont plus du ressort de l'entreprise mais de la Société, des individus et de leur bon vouloir, de leur capacité à se mobiliser, à gérer... Notre propos n'est pas ici de minimiser le rôle des dimensions intra ou interindividuelles dans l'intégration des nouveaux; toutefois signalons avec les tenants d'une conception du sujet proactif dans le processus d'insertion (Almudever, \& Le Blanc, 2006) que cette tendance à la psychologisation des échecs «peut masquer un ancrage organisationnel de certaines difficultés » (p. 146). Ce point nous semble essentiel et nécessite d'être discuté plus amplement. Les entreprises demandent aux nouveaux et anciens de coopérer, de s'entraider, d'échanger des informations comme si les changements apportés par les outils de GRH (la multiplication de l'intérim, la diversification des offres de formation, les dispositifs de fidélisation, la gestion du personnel par les compétences...) n'avaient pas d'inci- 
dence sur les possibilités réelles de coopérer, de s'entraider et de transmettre. Comme si l'utilisation conjointe de ces outils sous-tendant des logiques d'actions antagonistes (embauche par intérim et dispositif de fidélisation par exemple) ne venait pas compromettre dans le travail toute coopération ou transmission entre personnes de générations différentes. Ces premières réflexions laissent entendre que si les motifs de l'emploi - d'avoir un emploi - ou les modalités d'accueil et d'accompagnement des nouveaux (acteurs ou objets de leur insertion) sont certes importants (Cohen-Scali, 2008), ils ne sont pas seuls à entrer en ligne de compte dans le souhait de rester ou de partir et que des questions de travail, de conditions de travail, peuvent aussi participer à ce choix.

Cette perspective permet d'éclairer autrement les tensions observées entre anciens et nouveaux qui pourraient être interprétées, à un premier niveau, comme un conflit de valeur où la question de la fidélisation, pour les anciens, se joue dans les preuves données de la fidélité du nouveau. Nous ne pouvons ignorer des différences entre générations. Beaud et Pialoux $(1999 ; 2001)$ avancent que le chômage et la précarité sont des expériences communes à de nombreux jeunes ouvriers depuis les années 90 et semblent avoir instauré une rupture avec les générations précédentes. Toutefois, les anciens que nous avons rencontrés sont souvent des quarantenaires, voire des trentenaires. Même si les stéréotypes classiques (le jeune irrespectueux et l'ancien ancré dans la tradition et l'autorité) peuvent s'appuyer sur des expériences vécues, Flamant (2005) propose de rester attentif au contexte dans lequel ils se cristallisent. Ces rapports entre anciens et nouveaux ont pu en effet s'inscrire dans des changements organisationnels qui ont alors des conséquences sur les relations entre groupes d'âges et/ou d'anciennetés. Notre étude n'a pas porté directement sur l'analyse des évolutions organisationnelles dans l'entreprise, mais nous avons recueilli quelques indicateurs permettant d'émettre des hypothèses. La question d'un renforcement des contraintes temporelles est revenue à plusieurs reprises dans les discours des anciens avec des conséquences pour soi-même, pour l'équipe et pour la transmission. Ce renforcement des contraintes de temps est un phénomène aujourd'hui reconnu comme étant significatif d'un processus d'intensification du travail dans tous les secteurs (Askenazy, Cartron, De Coninck, \& Gollac, 2006). Outre la persistance des contraintes physiques et temporelles du travail que nous montre la dernière enquête nationale sur les conditions de travail (Bué, Coutrot, Hamon-Cholet, \& Vinck, 2007), l'augmentation des types de contraintes à prendre en compte (de quantité et de qualité, de contrôle, de délai...) - et parfois contradictoires entre elles - débouche sur des situations de débordement dans le travail où les salariés souffrent individuellement de ne pas pouvoir bien faire leur travail, sans être en situation d'en débattre avec leurs collègues (Théry, 2006). La fragilisation, voire la disparition, des collectifs à laquelle cette intensification conduit, est peu propice à une construction efficiente de l'expérience à tous les âges. Quand le manque d'effectif conduit à des constitutions d'équipes où seul un ancien est présent, comme nous l'avons vu, comment assurer la formation des nouveaux? Le deuxième indicateur que nous avons pu relever concerne les modes d'insertion passés et actuels des nouveaux dans les équipes. En dehors même du phénomène d'intérim qui laisse planer le doute - tant pour les anciens que pour les nouveaux - sur la présence durable des intérimaires, les rares situations où nous avons relevé une prise en charge des nouveaux organisée par le chef de chantier se faisait sur le principe de la rotation sur plusieurs postes et dans plusieurs équipes, afin d'identifier les capacités de polyvalence des nouveaux. Ce mode d'organisation se heurte souvent au mode antérieur, basé à l'inverse sur la reconnaissance de la spécialisation comme signe d'expertise (Gaudart, 2003): la polyvalence est au contraire le signe que l'opérateur n'a pas encore trouvé sa place. Les formes de compagnonnage, qui se sont construites sur ce mode organisationnel antérieur, supposent d'avoir du temps devant soi et l'appréciation de la valeur du nouveau ne constitue qu'une première étape.

\section{2.- La pratique professionnelle au centre de la transmission}

Par ailleurs, nos résultats mettent en avant - dispositifs d'accueil ou non - la disparition, ou pour le moins la mise à la marge, dans la transmission de ce que nous nommerons « le geste professionnel », pourtant essentiel à la construction de la pratique. Cette éviction du geste professionnel amène 
directement les nouveaux à prendre en charge leur apprentissage dans des conditions plus ou moins favorables pour leur santé physique et psychique et pour construire une pratique professionnelle sur le long terme. Elle s'observe à la fois dans l'organisation des dispositifs de formation et, dans une certaine mesure, au niveau de l'encadrement et des anciens eux-mêmes; elle prend sa source dans une opposition entre « valeurs » et « techniques ». Dans la formation de coffreur-bancheur, la technique du métier est considérée par les organisateurs comme pouvant se transmettre en dehors de tout contexte de travail: elle est transversale aux trois branches (le Bâtiment, les TP canalisation et le Génie civil) et peut se maîtriser en centre de formation sur une courte durée (deux semaines), en dehors du contexte du chantier. De ce fait, ce sont d'autres critères qui sont retenus pour le recrutement et qui renvoient à la « valeur » des candidats, prise dans une acception restrictive de l'étymologie du terme, i.e. à leur courage et leur vaillance, à leur force physique et morale (Rey, 2000). Le fait d'une part de réduire la valeur à des capacités individuelles et de dissocier, d'autre part, technique et valeur en considérant qu'à travers l'apprentissage d'une technique, on ne peut apprendre et transmettre des valeurs, pose problème. Cela conduit à sous-estimer le temps nécessaire à l'apprentissage qui n'est pas une simple reproduction du geste technique, mais une appropriation qui tient compte de ses propres caractéristiques (Chassaing, 2006). Cela conduit également à négliger toutes les phases d'observation et de tâtonnement dans l'acquisition d'une pratique professionnelle qui permettent aux apprenants de passer du statut de "participants périphériques légitimes » à celui d'anciens (Lave, 1991). Cela conduit enfin à ignorer qu'avec la dimension technique se transmettent, se construisent et se reconstruisent des valeurs de métiers constitutives d'un genre professionnel; ce que Clot, Fernandez et Scheller (2007) évoquent en ces termes: « [...] à la manière de Baktine (1978), on peut conclure que le geste ne se transmet pas comme un ballon qui rebondirait de sujet en sujet et même de génération en génération. En fait le geste au travers duquel se réalise un genre de métier ne se transmet pas, au sens strict. Il dure et perdure sous la forme d'une évolution ininterrompue. Il peut se perdre aussi. Quoi qu'il en soit, personne ne reçoit en partage un geste prêt-à-porter» (p. 133). C'est ici que devient alors possible la transformation du savoir-faire technique en geste professionnel, quand il prend en compte «soi » et « autrui ». Chassaing (2006) en tire les conséquences en matière de conception de dispositifs de formation, à partir du développement du concept de "gestuelles ». Il renvoie aux principes qui sous-tendent le geste et qui intègrent l'usage du corps. Ce sont des ensembles structurés et préalablement constitués qui forment des sortes d'armatures, relativement stables à court terme et utilisables dans différentes situations. Or, ces principes, essentiels à la construction d'une expérience professionnelle, ne sont pas directement observables et sont difficilement énonçables. De fait, les dispositifs de formation doivent s'appuyer sur des temps de mutualisation des pratiques qui permettraient de restituer le caractère complexe d'une gestuelle en mettant à jour les principes qui la soustendent, en la rendant moins « compliquée » en favorisant une structuration des savoirs (Savoyant, 2006). Ces principes permettent d'anticiper, de prendre des micro-décisions, de gérer la variabilité de la situation, de réduire la pénibilité du travail dans la phase d'acquisition. Ils deviennent une ressource pour comprendre et agir, diagnostiquer et construire l'action. Ils comprennent à la fois des aspects perceptifs et cognitifs que bien souvent les formations tendent à omettre (Mayen, 2006). Ils offriraient une alternative à l'enseignement du « bon geste » en amenant à découvrir, ou redécouvrir la pluralité des modes opératoires, leurs fondements, et donc leur légitimité.

Enfin, nous avons vu que l'encadrement de proximité joue également un rôle spécifique dans l'accueil des nouveaux, intérimaires comme apprentis, au regard des conditions de sécurité. Il semble que ce soit la première mission - et parfois l'unique - qu'ils s'assignent. Montrer les risques du chantier ne peut manquer de légitimité, mais en dehors de tout lien avec l'activité, cela peut mettre les nouveaux dans une posture intenable, entre transgression des règles de sécurité ou travail inachevé et «mal » réalisé. Comment planter un clou ? Nous l'avons vu: le nouveau s'y prenant mal risque de se blesser sans pouvoir réussir. L'ancien lui signale pourquoi il n'y arrive pas, sans lui expliquer comment faire; le chef d'équipe porte un jugement négatif sur ses capacités, lui demande de remettre son casque que le nouveau avait enlevé parce que gênant et l'envoie sur une autre tâche. En échos à ce mode de transmission partiel, autoritaire et unilatéral, nous proposons ici trois pistes de réflexion: 
- Le sentiment d'insécurité généré par le chantier peut conduire à un sentiment permanent de peur qui peut amener les nouveaux à relativiser l'intérêt d'apprendre le métier (Santos, \& Lacomblez, 2007) et les faire fuir. Si les idéologies défensives de métier que se sont construits les plus anciens leur permettent de tenir (Cru, 1995) qu'en est-il alors de leur transmission et de leur appropriation dans un tel contexte? Est-ce possible et à quelles conditions ? Est-ce souhaitable?

- Les déterminants de la transmission ne relèvent pas uniquement des conditions organisationnelles du travail et de la formation, mais renvoient aussi aux modalités pédagogiques à mettre en œuvre, aux moyens dont disposent les plus anciens pour mettre à jour et transmettre leur pratique professionnelle. Si nous partons du postulat que « transmettre » ne va pas de soi, se pose alors la question de la formation des tuteurs et notamment de la place et du rôle accordés à leur expérience dans l'apprentissage de l'acte de transmettre (Delgoulet, 2001 ; Cau-Bareille, Delgoulet, \& Gaudart, 2006).

- Les dispositifs d'accueil et de formation, censés fidéliser les nouveaux, passent à côté des enjeux de réciprocité et de reconnaissance mutuelle en privilégiant un sens à l'interaction (du tuteur au novice). Comment alors, compte tenu des conditions de travail effectives, permettre aux travailleurs, nouveaux et anciens, de devenir « des protagonistes de débats » (Lacomblez, 2007)?

\section{RÉFÉRENCES}

Aldeghi, I., \& Cohen-Scali, V. (2005). Orientation et professionnalisation des jeunes dans le secteur $d u$ bâtiment. Cahier de Recherche n ${ }^{\circ} 219$, Paris: Centre de Recherche pour l'Etude et l'Observation des Conditions de Vie.

Alexandre-Bailly, F., Gautié, J., Guillemard, A.-M., \& Jolivet, A. (2004). Gestion des âges et rapports intergénérationnels dans les grandes entreprises : études de cas. Partie II du Rapport de l'ACI "Travail, temps, trajectoires et transitions", juin, 264p.

Almudever, B., \& Le Blanc, A. (2006). Comment faciliter l'insertion des nouveaux recrutés dans des organisations du travail ? In C.Lévy-Leboyer, C. Louche, \& J.P. Rolland (Eds.), Ressources humaines : les apports de la psychologie du travail (pp. 125-148). Paris: Editions d'Organisation, vol 1.

Askenazy, P., Cartron, D., Coninck (De), F., \& Gollac, M. (Eds.) (2006). Organisation et intensité du travail. Toulouse: Octarès (Coll. Le travail en débats).

Beaud, S., \& Pialoux, M. (1999). Retour sur la condition ouvrière. Paris: Fayard.

Beaud, S., \& Pialoux, M. (2001). Travail, école: changements dans les rapports entre générations dans la région de Sochaux-Montbéliard. Actes du séminaire CREAPT, Générations et activité de travail (pp. 115134). Paris: EPHE-CREAPT (Coll. Cahiers du CREAPT).

Berthet, M., \& Cru, D. (2003). Travail prescrit, travail réel et santé au travail - De nouveaux modes d'intervention ergonomique? Travail et Emploi, 96, 85-96.

Blanchet, D. (2002). Le vieillissement de la population active: ampleur et incidence. Économie et Statistiques, 355-356, 123-138.

Bourgeois, F., \& Hubault, F. (2005). De la biomécanique à la revalorisation du travail, l'analyse du geste dans toutes ses dimensions. @ctivités, 2 (1). http://www.activites.org

Bué, J., Coutrot, T., Hamon-Cholet, S., \& Vinck, L. (2007) Conditions de travail: une pause dans l'intensification du travail. Premières Informations et Premières Synthèses, DARES, $\mathrm{n}^{\circ} 01.2$. www.travail. gouv.fr/etudes-recherche-statistiques/etudes-recherche/publications-dares/98.html

Cau-Bareille, D., Delgoulet, C., \& Gaudart, C. (2006). When learning difficulties and specificities of older workers stand to show training deficiencies. Proceedings IEA 2006 Congress "Meeting Diversity in Ergonomics", 10 to 15 July: Maastricht (The Netherlands), [Cédérom] Elsevier. 
Chardon, O., Estrade, M.-A., \& Toutlemonde, F. (2005). Les métiers en 2015 : l'impact du départ des générations du baby-boom. Premières Informations et Premières Synthèses, DARES, $\mathrm{n}^{\circ}$ 50.1. www. travail.gouv.fr/etudes-recherche-statistiques/etudes-recherche/publications-dares/98.html

Chardon, O., \& Viney, X. (2003). Le retournement de conjoncture en 2001-2002: que sont devenues les « difficultés de recrutement»? Premières Informations et Premières Synthèses, DARES, n 19.2. www. travail.gouv.fr/etudes-recherche-statistiques/etudes-recherche/publications-dares/98.html

Chassaing, K. (2006). Elaboration, structuration et réalisation des gestuelles de travail: les gestes dans l'assemblage automobile, et dans le coffrage des ponts d'autoroute. Thèse d'Ergonomie, Paris: Laboratoire d'Ergonomie, CNAM.

Clot, Y. (1999). La fonction psychologique du travail. Paris: PUF (Coll. Travail Humain).

Clot, Y., \& Faïta, D. (2000). Genres et styles en analyse du travail. Concepts et méthodes. Travailler, 4, 7-42.

Clot, Y., Fernandez, G., \& Scheller, L. (2007). Le geste de métier: problèmes de la transmission. Psychologie de l'interaction, 23-24, 109-138.

Cloutier, E., Lefebvre, S., Ledoux, E., Chatigny, C., \& St-Jacques, Y. (2002). Enjeux de santé et de sécurité au travail dans la transmission des savoirs professionnels : le cas des usineurs et des cuisiniers. Rapport n 316, Montréal: IRSST. http://www.irsst.qc.ca/fr/ publicationirssts par champ 70.html

Cohen-Scali, V. (2008). Accompagnement en entreprise et intentions liées à la carrière chez les apprentis du bâtiment. Pratiques Psychologiques, 14, 147-160.

Cru, D. (1995). Travail et santé mentale. Analyses et Documents Economiques, 64, 42-46.

Delgoulet, C. (2001). La construction des liens entre situations de travail et situations d'apprentissage dans la formation professionnelle. PISTES, 3(2). http://www.unites.uqam.ca/pistes/.

Dubré, J.-Y., Touranchet, A., Thérond-Hamidou, M., Monfort, C., \& Derriennic, F. (1996). Age, douleurs ostéo-articulaires et sélections au travail parmi les ouvriers du Bâtiment et des Travaux Publics. In F. Derriennic, A. Touranchet, \& S. Volkoff (Eds.), Age, travail, santé, études sur les salariés âgés de 37 à 52 ans (pp. 245-274). Paris: INSERM.

Duc, M. (2002). Le Travail en chantier. Toulouse: Octarès (Coll. Travail et Activité Humaine).

Flamant, N. (2005). Conflit de générations ou conflit d'organisation? Un train peut en cacher un autre... Sociologie du Travail, 47(2), 223-244.

Gaudart, C. (2003) La baisse de la polyvalence avec l'âge : question de vieillissement, d'expérience, de génération? PISTES, 5(2). http://www.pistes.uqam.ca/

Gaudart, C., Molinié, A.F., \& Pueyo, V. (2006). Du vieillissement à la diversité des âges au travail Questions pour l'ergonomie. In Actes du XXXXIème congrès de la SELF, Ergonomie et Santé au Travail (pp.471-47). Toulouse@: Octarès (Coll. Le travail en débats)

Hansez, I., Bertrand, F., De Keyzer, V., \& Férée, F. (2005). Fin de carrière des enseignants : vers une explication du stress et des retraites prématurées. Le Travail Humain, 68(3), 193-223.

Harmon-Cholet, S., \& Sandret, N. (2007). Accidents et conditions de travail. Premières Informations et Premières Synthèses, DARES, $\mathrm{n}^{\circ}$ 31.2. www.travail.gouv.fr/etudes-recherche-statistiques/etudesrecherche/publications-dares/98.html

Lacomblez, M. (2007). Réactions à l'exposé de C. Delgoulet et C. Gaudart «Ages et apprentissage », Actes du séminaire CREAPT, Age, santé, travail: quelles évolutions? 15 ans de travaux du CREAPT n³7. Noisy-Le-Grand: CEE (Coll. Rapport de Recherche). http://www.cee-recherche.fr/fr/rapports.htm

Lainé, F. (2005). De la spécialité de formation au métier: le cas du bâtiment, de l'hôtellerie-restaurationalimentation et du commerce. Economie et Statistique, 388-389, 145-169.

Lasfargues, G. (2005). Départs en retraite et «travaux pénibles »-L'usage des connaissances scientifiques sur le travail et ses risques à long terme pour la santé. Rapport de recherche $\mathrm{n}^{\circ} 19$, Noisy-Le-Grand: CEE/CREAPT. http://www.cee-recherche.fr/fr/rapports.htm 
Lave, J. (1991). Acquisition des savoirs et pratiques de groupes. Sociologie et Sociétés, 13(1), 145-162.

Mayen, P. (2006). Transmission en situation et didactique professionnelle. Actes du séminaire CREAPT, Transmission des savoirs professionnels en Entreprise, $n^{\circ} 35$, Noisy-Le-Grand: CEE (Coll. Rapport de Recherche), 111-124. . http://www.cee-recherche.fr/fr/rapports.htm

Merlino, L.A., Rosecrance, J.A., Anton, D., \& Cook, T.M. (2003). Symptoms of Musculoskeletal Didorders among apprentice construction workers. Applied Occupational and Environmental Hygiene, 18(1), 57-64.

Molinié, A.F. (2001). Les générations au travail : cadrage démographique et enjeux pour l'activité. Actes du séminaire CREAPT, Générations et activité de travail (pp. 1-13). Paris: EPHE-CREAPT (Coll. Cahiers du CREAPT).

Molinié, A.F. (2003). Les enjeux démographiques et les parcours professionnels différenciés. Session Spéciale du XXXXIIéme Congrès de la SELF, «Rester plus longtemps, dans quel travail ? L'ergonomie et la question de la durée de la vie active ». http://www.ergonomie-self.org/self2003/session2003.pdf

Molinié, A.-F., (2005a). Se sentir capable de rester dans son emploi jusqu'à la retraite ? Pistes, 7(1). http:// www.pistes.uqam.ca/

Molinié, A.-F. (2005b). Enquête « Santé et Vie Professionnelle après 50 ans »-Résultats par secteur d'activité. Rapport de Recherche ${ }^{\circ}$ 26. Noisy-Le-Grand: CEE/CREAPT. http://www.cee-recherche.fr/fr/ rapports.htm

Pichault, F., \& Nizet, J. (2000). Les pratiques de gestion des ressources humaines. Approches contingente et politique. Paris: Seuil.

Rey, A. (Ed.) (2000). Dictionnaire historique de la langue française. Paris: Dictionnaire Le Robert Editions.

Riccardis (De), N. (2007). L'intérim en 2006: une croissance toujours soutenue. Premières Synthèses et Premières Informations, DARES, ${ }^{\circ}$ 30.2. www.travail.gouv.fr/etudes-recherche-statistiques/etudesrecherche/publications-dares/98.html

Rousselet, J. (1987). Attitudes des jeunes face au travail et au non travail. In C. Lévy-Leboyer, \& J.-C., Sperandio (Eds). Traité de psychologie du travail (pp. 57-76). Paris: PUF.

Sanchez, R. (2007). L'apprentissage en 2005 : une nouvelle hausse des entrées. Premières Synthèses et Premières Synthèses, DARES, n 13.3. www.travail.gouv.fr/etudes-recherche-statistiques/etudesrecherche/publications-dares/98.html

Santos, M., \& Lacomblez, M. (2007). Que fait la peur d'apprendre dans la zone prochaine de développement? @ctivités, 4(2), 16-29, http://www.activites.org/v4n2.pdf

Savoyant, A. (2006). Les problèmes de la transmission des savoirs professionnels en entreprise. Actes $d u$ séminaire Vieillissement Travail : "Transmission des savoirs professionnels en Entreprise », $\mathrm{n}^{\circ} 35$, Noisy-Le-Grand: CEE (Coll. Rapport de Recherche), 9-19. http://www.cee-recherche.fr/fr/rapports.htm

Six, F. (1997). La préparation du travail - Un enjeu pour la sécurité et la valorisation des compétences, Rapport «Etudes et Expérimentations Chantier 2000 », Lille: GERN.

Six, F. (1999). De la prescription à la préparation du travail-Apports de l'ergonomie à la prévention et à l'organisation du travail sur les chantiers du BTP. Habilitation à Diriger des Recherches, Lille 3: Université Charles de Gaulle.

Stroobants, M. (2003). L'individualisation des relations salariales par la gestion des compétences. In A. Dupray, C. Guitton, \& S. Monchatre (Eds.), Réfléchir la compétence. Approches sociologiques, juridiques, économiques d'une pratique gestionnaire (pp. 43-52). Toulouse: Octarès.

Théry, L. (Ed.) (2006). Le Travail intenable - Résister collectivement à l’intensification du travail. Paris: La Découverte (Coll. Entreprise Société). 


\section{RÉSUMÉ}

Les départs à la retraite actuels et à venir des baby-boomers donnent l'opportunité à des entreprises de renouveler leur main-d'œuvre. Elles sont aussi nombreuses à éprouver des difficultés à conserver les nouveaux embauchés, pour la plupart jeunes, malgré un contexte de chômage. Plusieurs facteurs peuvent entrer en ligne de compte et complexifient un problème souvent traité en termes de fidélisation des nouveaux, de conflit de générations et de représentations. Cet article propose une analyse de cette situation à partir d'une approche ergonomique, dans une grande entreprise du BTP. Malgré la diversité de statuts de «nouveaux » et la multiplicité des dispositifs de fidélisation par l'accueil et la formation, cette étude montre que le « geste professionnel » n'est en fait pas au centre des préoccupations de la GRH, des organisateurs au niveau des chantiers et - dans une certaine mesure - des anciens eux-mêmes. Cette absence débouche sur des formes d'apprentissage opportuniste mises en œuvre par les nouveaux ainsi mis à l'épreuve qui questionnent ces pratiques de fidélisation dans leur forme et leur contenu.

\section{MoTS CLÉS}

Transmission inter-générationnelle, fidélisation, apprentissage, geste professionnel, BTP.

\section{RESUMEN}

La fidelización de los nuevos empleados en una empresa constructora : Enfoque ergonómico de los desafíos y de los determinantes. La ingreso actual y por venir a la jubilación de los baby boomers ofrece a las empresas una oportunidad de renovación de su mano de obra. Asimismo, son numerosas aquellas que muestran dificultades para retener al personal recientemente contratado, en su mayoría jóvenes, a pesar del contexto de desocupación. Varios factores permiten complejizar un problema, a menudo tratado en términos de fidelización de los nuevos empleados, de generaciones y de representaciones. Este artículo propone un análisis de esta situación a partir de un enfoque ergonómico, en una gran empresa constructora. A pesar de la diversidad de categorías de los "nuevos empleados" y de la multiplicidad de dispositivos de fidelización vía el reclutamiento y la formación, este estudio muestra que el « gesto profesional » no es la principal preocupación de la Gerencia de Recursos Humanos, de los organizadores a nivel del obrador y, en cierta medida, de los trabajadores más experimentados. Esta ausencia desemboca en formas de aprendizaje oportunista desplegadas por los nuevos empleados puestos a prueba de este modo, que cuestionan las prácticas de fidelización, tanto en su forma como en su contenido.

\section{Palabras ClaVe}

Transmisión inter-generacional, fidelización, aprendizaje, gesto profesional, Construcción

\section{RÉFÉRENCEMENT}

Gaudart, C., Delgoulet, C., \& Chassaing, K. (2008). La fidélisation de nouveaux dans une entreprise du BTP: Approche ergonomique des enjeux et des déterminants. Activités, 5 (2) pp. 2-24, http://www.activites.org/ v5n2/v5n2.pdf

Article soumis le 14 novembre 2007 accepté pour publication le 13 juin 2008 